\title{
Stage III Gestational Trophoblastic Tumor AJCC v7
}

National Cancer Institute

\section{Source}

National Cancer Institute. Stage III Gestational Trophoblastic Tumor A/CC V7. NCI

Thesaurus. Code C87156.

Stage III includes: (Any T, M1a, Risk factors: unknown); IIIA (Any T, M1a, Risk factors: low risk); IIIB (Any T, M1a, Risk factors: high risk). M1a: Lung metastasis. (AJCC 7th ed.) 